\title{
All-polymer Planar Photonic Crystals as an Innovative Tool for the Analysis of Air
}

\author{
Paola Lova, ${ }^{1 *}$ Heba Megahd, ${ }^{1}$ Davide Comoretto ${ }^{1}$ \\ ${ }^{1}$ Dipartimento di Chimica e Chimica Industriale, Università di Genova, 16146 Genova, Italy.
}

\begin{abstract}
The possibility to evaluate the molecular diffusivity in polymer thin films used for packaging and device encapsulation directly in-situ would represent a paradigm changer in the assesment of barrier properties and of air quality. Indeed, employing the packaging itself as a smart sensor could lead to waste reduction and mitigate food poisoning effects. In this work, we demonstrate a new technique that exploits simple UV-Vis reflectance spectroscopy to identify the kinetic of diffusion of small molecules in the vapor phase through polymer thin films and polymer multilayered structures. The new method allows then to assess the presence of the analyte in air and its diffusion coefficient in agreement with gravimetric data reported in literature.
\end{abstract}

\section{Introduction}

Diffusion coefficient of vapor molecules through polymeric films is usually determined by methods such as gravimetry ${ }^{[1]}$ and pressure decay,[2] or by optical techniques like microscopy, ${ }^{[3]}$ infrared spectroscopy,[4] and refractive index variations.[5] These techniques remained substantially unchanged for the last decades and currently do not allow fast and in-situ measurements. Moreover, when only a small amount of polymer is available more sophisticated approaches including NMR, and neutron reflectometry are required. [6-8] Assessing diffusive processes in polymer used for packaging materials is then a serious issue. Indeed, most polymers can absorb large amount of solvents, degradation byproducts, pollutants, and moisture promoting additive and plasticizer migration and release. This phenomenon can reduce the barrier properties of the packaging itself and contaminate food.[9] As a consequence, low-cost, and simple methods for the characterization of packaging properties is interesting from technological and safety point of views. To this end, polymer distributed Bragg reflectors (DBRs) made of thin films have shown large pollutants absorption that result in an optical response with kinetics depending on the pollutant themselves (Fig. 1) .[10-20] While these, and other solution processed structures are commonly used for light control applications such as for switchers,[21,22] photovoltaics,[23] lighting,[21,24-27] and lasing,[11,16,28,29] their use as sensor is a recent discovery that is opening new prospects for photonics and new methods applicable also to non-structured polymer films.[11]

Fig. 1 schematizes a DBRs made of media with different refractive index alternated periodically to generate a dielectric lattice. When light impinges on these structures, the formation of frequency regions where light propagation is forbidden generates photonic band gaps (PBGs) that can be measured via simple UVVis reflectance[30] as maxima in the DBR spectrum (Fig. 1b, green line). The background of the spectrum of these structures is instead dominated by an interference pattern, that is also typical of transparent thin film with small surface roughness. The properties of these spectral features are subjected to the modifications of the lattice components refractive index and thicknesses.[31] Consequently, perturbing these parameters affects the entire spectrum, and the spectral variations can then be related to stimuli such as pressure variations,[32] chemical analytes, $[14,15,33]$ and $\mathrm{pH} .[34]$ The red line of Fig. 1c displays the typical response of a polymer DBR spectrum after intercalation of an analyte. Such variations usually consist in the red shift of the PBG due to the swelling of the polymer components. Notice that, in this case, the spectral position of the PBG shifted from the green region of the spectrum to the red one, allowing a simple colorimetric response.

Interestingly, the kinetics of these optical responses allows to retrieve information on the polymer-analyte interactions. For instance, optical sorption curves can be obtained tracing the PBG during exposure to analytes. Such curves can then be treated as gravimetric data[35] obtaining values of molecular diffusion coefficients comparable with those reported in literature.[10,35] This is possible thanks to the chemico-physical interactions between polymer and molecules that rules their diffusion, and also allow to identify the molecular specie without using any chemical target.[10,20] Here, we demonstrate that such method can be applied to the interference pattern of DBR, thus making the method suitable to any polymer thin film. For this purpose we expose a DBR made of cellulose acetate and polystyrene

\footnotetext{
* Corresponding author: paola.lova@edu.unige.it
} 
to methanol vapor and extract optical sorption curves from both the PBG and the interference pattern.
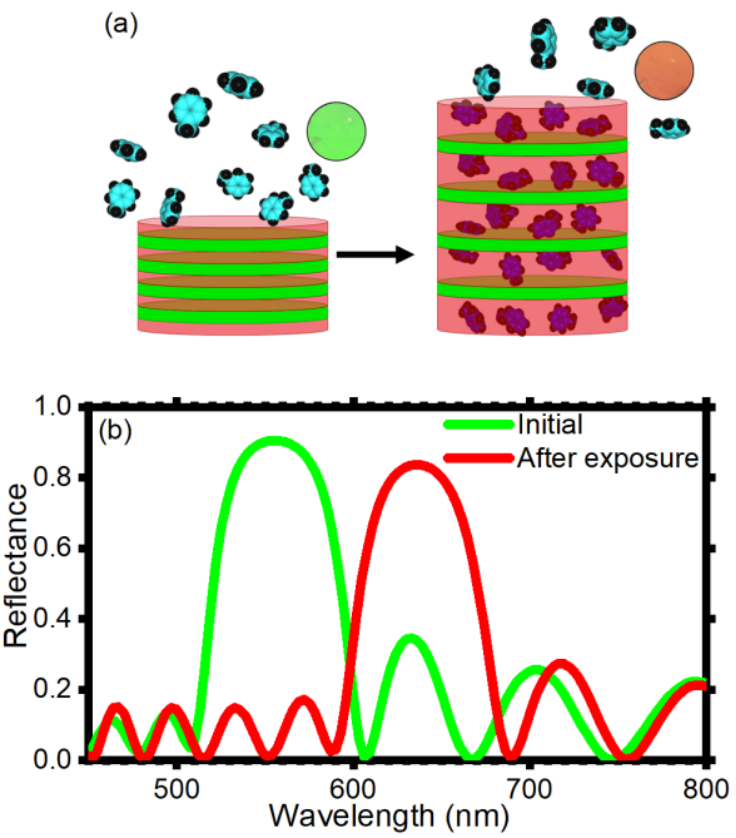

Fig. 1. a) Scheme of the behavior of a DBR exposed to vapor molecules. b) Typical optical response of a DBR (green line), and the same sample after vapor exposure (red line).

\section{Experimental Methods}

The DBRs were spun-cast from cellulose acetate (CA) solution in diacetone alcohol and polystyrene (PS) in toluene as described in previous works.[14,15] For all the samples reflectance data were collected during exposure to pure methanol vapor as previously described.[14]

\section{Results and Discussion}

To demonstrate that this simple method can be applied to unstructured polymer films, Fig. 2 reports the optical response for a DBR made of 10 bilayers of PS and CA with total thickness of about $4 \mu \mathrm{m}$ and compare it with the response. In Fig. 2a, the DBR PBG is initially positioned at about $840 \mathrm{~nm}$ before the exposure to methanol vapor, after which it undergoes a remarkable shift to $950 \mathrm{~nm}$. Interestingly, also the interference pattern shifts during the exposure. Indeed, such pattern arises from reflection phenomena occurring at the external DBR interfaces, and then it reflects the global thickness variations occurring in the sample. Comparing the normalized spectral positions of the PBG and of three relative maxima of the interference, we notice that the retrieved curves are perfectly superimposable (Fig. $2 b$ ). Indeed, from these curves identical effective diffusion coefficients, that is $\mathrm{D}=2.5^{*} 10^{-8} \mathrm{~cm}^{2} / \mathrm{s}$ in agreement with literature gravimetric data is obtained. ${ }^{25,37}$ Such value demonstrated that the process can be potentially applied to any unstructured polymeric film showing an interference pattern. Notice that the nice similarity between the curves allow to perform the measurements in any part of the UV-Vis spectrum making the method also suitable for non-transparent materials.
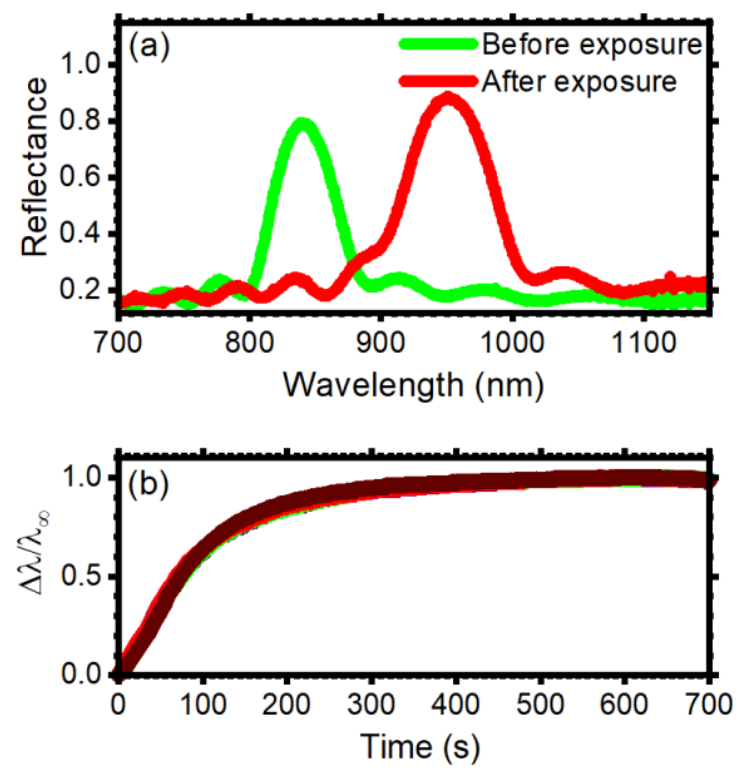

Fig. 2. Optical response of PS:CA DBR to exposure to methanol vapors. (a) Spectra collected before (green line) and after (red line line) the exposure. (b) Optical sorption curves extracted for the sample.

\section{Conclusions}

We demonstrated that the simple UV-Vis analysis allows of DBR interference pattern allows to assess the presence of vapor pollutant in the vapor phase and to retrieve optical sorption curves. The curves are retrieved tracing the maxima of the interference pattern during exposure to the vapor and allows to easily determines molecular diffusion coefficient in agreement with gravimetric data retrieved from literature using classical data analysis.

\section{References}

1. Berens, A.R.; Hopfenberg, H.B., J. Membr. Sci., 10, 283-303, (1982).

2. Flaconneche, B.; Martin, J.; Klopffer, M., Oil Gas Sci. Technol., 56, 245-259, (2001).

3. Mráček, A., Int. J. Mol. Sci., 11, 532-543, (2010).

4. Ferrari, M.-C.; Catalano, J.; Giacinti Baschetti, M.; De Angelis, M.G.; Sarti, G.C., Macromolecules, 45, 1901-1912, (2012).

5. Gaffney, C.; Chau, C.-K., Am. J. Phys., 69, 821825, (2001).

6. Xu, L.; Selin, V.; Zhuk, A.; Ankner, J.F.; Sukhishvili, S.A., ACS Macro Lett., 2, 865-868, (2013).

7. Meurer, B.; Weill, G., Macromol. Chem. Phys., 209, 212-219, (2008). 
8. Meier, R.; Herrmann, A.; Kresse, B.; Privalov, A.F.; Kruk, D.; Fujara, F.; Rössler, E.A., ACS Macro Lett., 2, 96-99, (2013).

9. Sharma, V.; Nani, D.; Kumar, R., Spectrochim. Acta, Part A, 206, 558-568, (2019).

10. Lova, P.; Manfredi, G.; Bastianini, C.; Mennucci, C.; Buatier de Mongeot, F.; Servida, A.; Comoretto, D., ACS Appl. Mater. Interfaces, 11, 16872-16880, (2019).

11. Lova, P.; Manfredi, G.; Comoretto, D., Adv. Opt. Mater., 6, 1800730-1800726, (2018).

12. Lova, P., Polymers, 10, 1161, (2018).

13. Lova, P.; Comoretto, D., AIP Conf. Proc., 1981, 020097, (2018).

14. Lova, P.; Bastianini, C.; Giusto, P.; Patrini, M.; Rizzo, P.; Guerra, G.; Iodice, M.; Soci, C.; Comoretto, D., ACS Appl. Mater. Interfaces, 8, 31941-31950, (2016).

15. Lova, P.; Manfredi, G.; Boarino, L.; Comite, A.; Laus, M.; Patrini, M.; Marabelli, F.; Soci, C.; Comoretto, D., ACS Photonics, 2, 537-543, (2015).

16. Lova, P.; Soci, C. Nanoimprint Lithography: Toward Polymer Photonic Crystals. In Organic and Hybrid Photonic Crystals, 1st ed.; Comoretto, D., Ed. Springer: Cham, 2015; Vol. 1, p 493.

17. Lova, P.; Manfredi, G.; Boarino, L.; Laus, M.; Urbinati, G.; Losco, T.; Marabelli, F.; Caratto, V.; Ferretti, M.; Castellano, M., et al., Phys. Status Solidi C, 12, 158-162, (2015).

18. Mönch, W.; Dehnert, J.; Prucker, O.; Rühe, J.; Zappe, H., Appl. Opt., 45, 4284-4290, (2006).

19. Mönch, W.; Dehnert, J.; Jaufmann, E.; Zappe, H., Appl. Phys. Lett., 89, 164104, (2006).

20. Gao, S.; Tang, X.; Langner, S.; Osvet, A.; Harrei $\beta$, C.; Barr, M.; Spiecker, E.; Bachmann, J.; Brabec, C.J.; Forberich, K., ACS Appl. Mater. Interfaces, 10, 36398-36406, (2018).

21. Paternò, G.M.; Iseppon, C.; D’Altri, A.; Fasanotti, C.; Merati, G.; Randi, M.; Desii, A.; Pogna, E.A.A.; Viola, D.; Cerullo, G., et al., Sci. Rep., 8, 3517, (2018).

22. Paternò, G.M.; Moscardi, L.; Kriegel, I.; Scotognella, F.; Lanzani, G., SPIE Proc., 8, 1-8, (2018).

23. Iasilli, G.; Francischello, R.; Lova, P.; Silvano, S.; Surace, A.; Pesce, G.; Alloisio, M.; Patrini, M.; Shimizu, M.; Comoretto, D., et al., Mater. Chem. Front., 3, 429-436, (2019).

24. Lova, P.; Giusto, P.; Stasio, F.D.; Manfredi, G.; Paternò, G.M.; Cortecchia, D.; Soci, C.; Comoretto, D., Nanoscale, 11, 8978-8983 (2019).

25. Lova, P.; Cortecchia, D.; S. Krishnamoorthy, H.N.; Giusto, P.; Bastianini, C.; Bruno, A.; Comoretto, D.; Soci, C., ACS Photonics, 5, 867-874, (2018).

26. Manfredi, G.; Lova, P.; Di Stasio, F.; Krahne, R.; Comoretto, D., ACS Photonics, 4, 1761-1769, (2017).

27. Lova, P.; Grande, V.; Manfredi, G.; Patrin, M.; Herbst, S.; Würthner, F.; Comoretto, D., Adv. Opt. Mater., 5, 1700523, (2017).
28. Manfredi, G.; Lova, P.; Di Stasio, F.; Rastogi, P.; Krahne, R.; Comoretto, D., RSC Advances, 8, 13026-13033, (2018).

29. Robbiano, V.; Paternò, G.M.; La Mattina, A.A.; Motti, S.G.; Lanzani, G.; Scotognella, F.; Barillaro, G., ACS Nano, 12, 4536-4544, (2018).

30. Comoretto, D. Organic and Hybrid Photonic Crystals. Springer: Cham, 2015.

31. Graham-Rowe, D., Nat. Photon., 3, 551-553, (2009).

32. Fang, Y.; Ni, Y.; Leo, S.-Y.; Taylor, C.; Basile, V.; Jiang, P., Nat. Comm., 6, 7416, (2015).

33. Lee, J.-H.; Koh, C.Y.; Singer, J.P.; Jeon, S.-J.; Maldovan, M.; Stein, O.; Thomas, E.L., Adv. Mater., 26, 532 - 569, (2013).

34. Wang, Z.; Zhang, J.; Wang, Z.; Shen, H.; Xie, J.; Li, Y.; Lin, L.; Yang, B., J. Mater. Chem. C, 1, 977983, (2013).

35. Crank, J. The Mathematics of Diffusion. 2nd ed.; Oxford university press: Bristol, 1975. 